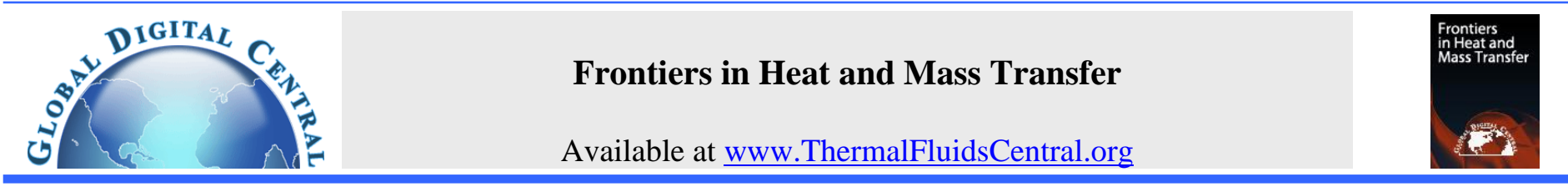

\title{
HEAT TRANSFER ANALYSIS FOR THE UNSTEADY UCM FLUID FLOW WITH HALL EFFECTS: THE TWO-PARAMETER LIE TRANSFORMATIONS
}

\author{
Muhammad Nazim Tufail ${ }^{\mathrm{a}}$, Musharafa Saleem ${ }^{\mathrm{a}, \mathrm{b}, *}$, Qasim Ali Chaudhry ${ }^{\mathrm{b}, \dagger}$ \\ ${ }^{a}$ Department of Mathematics, University of Management and Technology Lahore, Sialkot Campus, 51310, Pakistan \\ ${ }^{\mathrm{b}}$ Department of Mathematics, University of Engineering and Technology, Lahore, 54890, Pakistan
}

\begin{abstract}
This methodology presented the unsteady three-dimensional laminar flow since Hall effects inducing the cross flow in $\widetilde{z}$-axis. The boundary layer and the low magnetic Reynolds number approximations are used to simplify the system of equations derived from the constitutive laws. The upper-convected Maxwell (UCM) fluid model used for Hall effects with unsteady heat transfer, which passed through the infinite stretching sheet. This flow model has intensified with the effects of magnetohydrodynamic (MHD), thermal radiation and heat generation-absorption. Here, we selected the two-parameter Lie scaling transformations to convert the highly non-linear partial differential equations (PDEs) to the ordinary differential equations (ODEs) which are studied numerically using the MATLAB bvp4c method. The main parameters are: Deborah number $D e_{u}$, Hartmann number $M_{u}$, Hall effects parameter $m_{u}$, Prandtl number $\operatorname{Pr}$, thermal radiation parameter $\delta_{u}$ and heat generation-absorption $Q_{u}$. Hall effects reduced the transport rate in the $\widetilde{x}$-axis but increased the transport rate in the $\widetilde{z}$-axis. On the other hand, the Hall parameter is extravagant to transport the internal energy of the system.
\end{abstract}

Keywords: Hall effects; UCM; Stretching sheet; Two-parameter Lie scaling; thermal radiation.

\section{INTRODUCTION}

The advent of non-Newtonian fluid dynamics is based on the Newton's law of viscosity theory, which is not appropriate for the analysis of all the rheological properties of the fluid. The non-Newtonian fluids were not examined by a single constitutive equation. There are many constitutive relationships for the discovery of the numerous properties of nonNewtonian fluids. In addition, non-Newtonian fluids are categorized into differential form, rate form and integral type. For reality, the major applications of non-Newtonian fluids in the fields of material production, the chemical industry and bioengineering can be described Huang et al. (2019). Examples include mercury amalgams in dental surgery, liquid metals, plastic extrusions, paper coating process, lubricants and different types of oils Ndlovu and Moitsheki (2018); Oosthuizen and Manna (2019). Stress relaxation effects are the main characteristics of the model of the rate type. Through this view, James Clerk Maxwell introduced a model that did not have the capacity to shear dependent viscosity and instead investigated the fluid elasticity.

Due to its large applications, Rajagopal and Gupta (1984) presented an accurate analysis of the non-Newtonian fluid that passed through an infinite porous plate. Sadeghy et al. (2005) demonstrated the flow of UCM to the Sakiadis. Jamil and Fetecau (2010) analyzed the UCM model between helical-flow coaxial cylinders. Mixed convection is used by Abbas et al. (2010) for the Maxwell fluid with stagnation point flow and stretch- ing sheet. Furthermore, the exact solutions are provided by Zheng et al. (2011) by choosing the Maxwell fluid model with an oscillatory channel. The UCM fluid model is used by Hayat et al. (2012) for moving surface with thermal transfer analysis. To extend this form of flow Abel et al. (2012) proposed a heat transfer study of the MHD flow for the UCM fluid model. In addition, Awais et al. (2014) advocated three-dimensional fluid flow for the UCM model. The natural convection used by Zhao et al. (2016) for the fractional Maxwell fluid model, which moves over the vertical plate. Nonlinear thermal radiation, non-uniform heat generationabsorption and fluid particle suspension effects are discussed by Gireesha et al. (2018) for mixed convective Maxwell fluid. In addition , there are numerous recent studies on the UCM fluid model Hussain et al. (2016); Mahanthesh et al. (2017); Bilal et al. (2018); Kashyap et al. (2019); Farooq et al. (2019). The MHD study is essential in order to improve the transport rate and the transport of internal energy within the system. The MHD effect plays a vital role in a variety of fields, such as astrophysics, solar storms, stellar dynamics, solar structures and galaxy studies Vijaya and Ramana Reddy (2020). In this view, the Hall effect occurred as a result of the Lorentz force's action. In the case of a conductor, a current is passed and a magnetic field is applied perpendicularly Dharmaiah et al. (2020). As a result, the electrical field potential of the system is developed in both directions of the current and the magnetic field. The current study of the Hall, first discussed by Edwin Hall and later provoked Hall et al. (1879) as a new action of magnetic currents. To extend this theory, many

\footnotetext{
${ }^{*}$ Corresponding author email:musharafa.saleem@ skt.umt.edu.pk

${ }^{\dagger}$ Current address:Department of Mathematics, College of Science, University of Háil, Saudi Arabia
} 
writers have presented a vast literature to identify the physics behind the MHD and the Hall effects ( Sato (1961), Jana and Datta (1977), Mandal and K. Mandal (1983), Nagy and Demendy (1995)). The Hall's effects on the flow of internal energy have become more interesting for researchers as it has various applications in all fields of science. It is Raju et al. (2011) which combines the effects of unstable MHD, Hall effects, continuous suction, oscillation and porous effects between two stretching sheets. Free convection is performed by Maripala and Naikoti (2015) with Hall effects and MHD flow for stretching sheets with viscous dissipation. The MHD free convection fluid flow of Seth et al. (2016) with Hall, thermal radiation and heat abortion effects is considered to be a moving vertical plate. Again, Sreedevi et al. (2016) studied the thermal, Hall and absorption effects of free convective flow through the stretching surface. Also, the exponential effects on the stretching sheet advocated by Srinivasacharya and Jagadeeshwar (2017) with the Hall and Joule heating effects. In addition, MHD fluid flow with specific effects, such as Hall, thermal radiation and heat generation-absorption, has been experienced by several researchers ( Reddy et al. (2018), Krishna and Jyothi (2018), VeeraKrishna et al. (2018), Padma and Suneetha (2018), 'Veera Krishna and Jyothi (2018)). Recently, ( Alkasasbeh et al. (2020), Zhou et al. (2020), Kaprawi et al. (2019), Khoshrouye Ghiasi and Saleh (2018)) presented various effects for the non-Newtonian fluid. Moreover, Tufail et al. (2020)applied group theoretic approached to analyzed the nonNewtonian fluid with oscillation effects. Three-dimensional flow was considered to be explored with Hall effects in the presence of an unsteady UCM fluid model. This fluid model is enhanced by thermal radiation and heat generation-absorption analysis. The law on the conservation of mass , momentum and energy modeled the flow of fluids. The resulting highly nonlinear PDEs are not easily addressed. So, two-parameter transformations of Lie scaling have been used. Two-parameter transformations transform the PDEs into ODEs by reducing the three independent variables to one independent variable. The converted ODEs have been fixed by bvp4c in MATLAB. The related parameters are provided in the graphs and the validation of the model studied in the literature.

\section{GEOMETRY OF THE PROBLEM}

The primary flow along the $\widetilde{x}$-axis as represented in (Figure 1 ) with vertical stretching sheet in upward direction and the secondary flow is along the $\widetilde{z}$-axis. $\widetilde{y}$-axis is normal to the stretching sheet which is lie in $\widetilde{x} \widetilde{z}$ plane. The velocity vector is $\widetilde{V}=(\widetilde{u}(\widetilde{t}, \widetilde{x}, \widetilde{y}, \widetilde{z}), \widetilde{v}(\widetilde{t}, \widetilde{x}, \widetilde{y}, \widetilde{z}), \widetilde{w}(\widetilde{t}, \widetilde{x}, \widetilde{y}, \widetilde{z}))$. The $H_{y}$ is the transverse magnetic field which is applied in parallel direction of $\mathrm{y}$-axis. Initially, for $t=0$ the surface and the fluid are at rest. The transport of the internal energy is maintained at $\widetilde{T}(\widetilde{t}, \widetilde{x}, \widetilde{y}, \widetilde{z})=\widetilde{T}_{u}$. But, for $\tilde{t}>0$ the surface is going to start move in its own plane with the velocity $\widetilde{u}(\widetilde{t}, \widetilde{x}, \widetilde{y}, \widetilde{z})=\frac{\widetilde{x}}{t}$ and the transfer of the internal energy is fixed with $\widetilde{T}(\tilde{t}, \tilde{x}, \tilde{y}, \tilde{z})=\widetilde{T}_{\infty}+\left(\widetilde{T}_{h}-\widetilde{T}_{\infty}\right) \frac{\widetilde{x}}{t^{2}}$. Choose the magnetic Reynolds number is very small then the induced magnetic field will become zero. Due to assumption of the infinite length of the $\tilde{y}$-axis, there is no flow variations in this direction. The imposition of the Hall current generated due to the strong magnetic field in the direction of the $\tilde{y}$-axis in Figure 1. After ignoring the ion slips, thermo electric, viscous and electrical dissipation effects, we used the constitutive laws, generalized Ohm's law and Maxwell's equations ( Harris (1977), Tie-Gang et al. (2009)) with Hall effects:

$$
\begin{gathered}
\nabla \cdot \mathbf{V}=0 \\
\rho \mathbf{a}_{u}=\nabla \cdot \mathbf{T}+\mathbf{J H}
\end{gathered}
$$

and

$$
\mathbf{J}+\frac{\omega_{e} \tau_{e}}{H_{y}}(\mathbf{J} \times \mathbf{H})=\sigma(\mathbf{E}+\mathbf{V} \times \mathbf{H})
$$

where $\mathbf{J}=\left(J_{x}, J_{y}, J_{z}\right)$ indicates the current density vector, $\mathbf{V}$ indicates the velocity vector, $\mathbf{E}$ indicates the electric field vector, $\mathbf{H}=\left(0, H_{y}, 0\right)$ indicates the magnetic induction vector, $\tau_{e}$ indicates the electron collision time, $\omega_{e}$ indicates cyclotron frequency of electron and $\sigma=\frac{e^{e} n_{e} \tau_{e}}{m_{e}}$ with

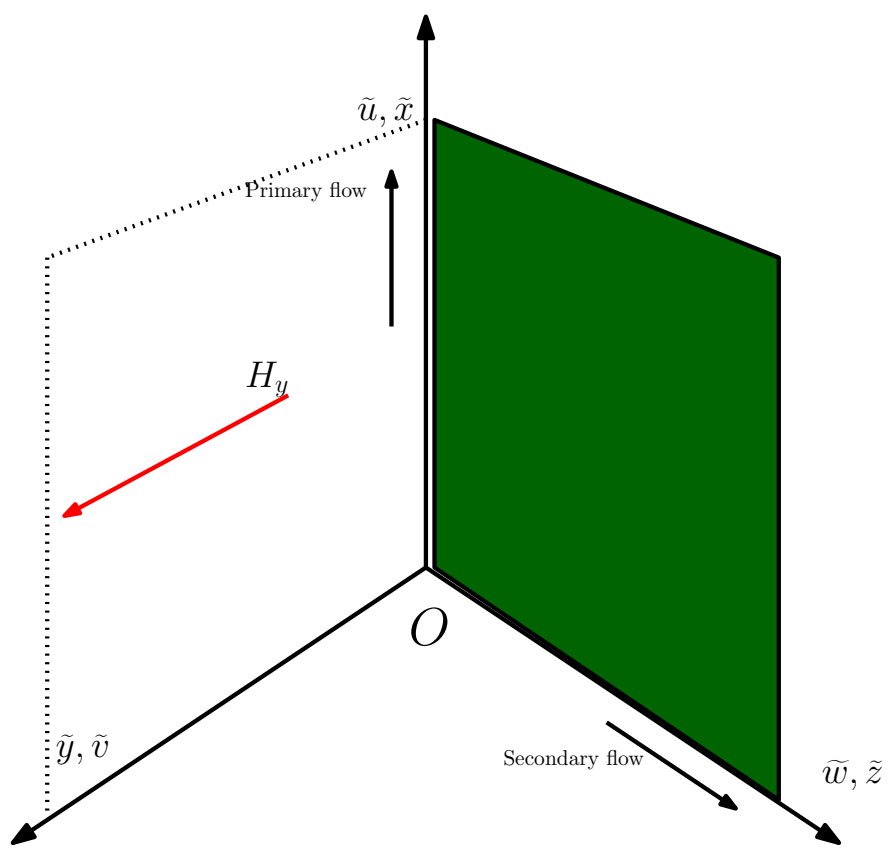

Fig. 1 Flow diagram due to Hall effects

$e$ indicates the charge of electron, $n_{e}$ indicates the number of density of electron, $m_{e}$ indicates the number of the electron) indicates the electrical conductivity. In Eq. (2)

$$
\mathbf{a}_{u}=\frac{d \mathbf{V}}{d t}=\frac{\partial V}{\partial t}+(\mathbf{V} . \nabla) \mathbf{V}
$$

and for UCM, the Cauchy stress tensor is

$$
\mathbf{T}=-p \mathbf{I}+\mathbf{S}
$$

and $\mathbf{S}$ is defined as

$$
\left(1+\lambda \frac{D}{D t}\right) \mathbf{S}=\mu \mathbf{A}_{1}
$$

where $\lambda$ indicates the relaxation time, $\mu$ indicates the dynamics viscosity and $\mathbf{A}_{1}$ indicates the first Rivlin-Ericksen tensor which is defined as

$$
\mathbf{A}_{1}=\mathbf{L}+\mathbf{L}^{T^{\prime}}, \mathbf{L}=\nabla \mathbf{V}
$$

Now, $\mathbf{S}$ indicated as a two rank tensor in Eq. (6), the relation can be written as

$$
\frac{D \mathbf{S}}{D t}=\frac{\partial \mathbf{S}}{\partial t}+(\mathbf{V} \cdot \nabla) \mathbf{S}-\mathbf{L S}-\mathbf{S L}^{T^{\prime}}
$$

where $T^{\prime}$ indicates the transpose vector and the momentum equation for $\mathrm{UCM}$ is

$$
\rho \mathbf{a}_{\mathbf{u}}=-\nabla p+\nabla \cdot \mathbf{S}
$$

applying $\left(1+\lambda \frac{D}{D t}\right)$ on both side of Eq. (9) then we get

$$
\begin{array}{r}
\rho\left(1+\lambda \frac{D}{D t}\right) \mathbf{a}_{\mathbf{u}}=-\left(1+\lambda \frac{D}{D t}\right) \nabla p+\left(1+\lambda \frac{D}{D t}\right) \nabla \cdot \mathbf{S}+ \\
\left(1+\lambda \frac{D}{D t}\right)(\mathbf{J} \times \mathbf{H})
\end{array}
$$

By following Tie-Gang et al. (2009)

$$
\frac{D}{D t}(\nabla \cdot)=\nabla \cdot\left(\frac{D}{D t}\right)
$$


using the above relation in Eq. (11), we arrived at

$$
\begin{array}{r}
\rho\left(1+\lambda \frac{D}{D t}\right) \mathbf{a}_{\mathbf{u}}=-\left(1+\lambda \frac{D}{D t}\right) \nabla p+\nabla \cdot\left(1+\lambda \frac{D}{D t}\right) \mathbf{S}+ \\
\left(1+\lambda \frac{D}{D t}\right)(\mathbf{J} \times \mathbf{H})=-\left(1+\lambda \frac{D}{D t}\right) \nabla p+\mu \nabla \cdot \mathbf{A}_{\mathbf{1}}+ \\
\left(1+\lambda \frac{D}{D t}\right)(\mathbf{J} \times \mathbf{H})
\end{array}
$$

After neglecting the pressure gradient term in Eq. (12) then the result is

$$
\rho\left(1+\lambda \frac{D}{D t}\right) \mathbf{a}_{\mathbf{u}}=\mu \nabla \cdot \mathbf{A}_{\mathbf{1}}+\left(1+\lambda \frac{D}{D t}\right)(\mathbf{J} \times \mathbf{H})
$$

For Eq. (3), the term $\mathbf{J}+\frac{\omega_{e} \tau_{e}}{H_{y}}(\mathbf{J} \times \mathbf{H})$ indicates the electric field in the moving frame. The law of conservation of current resulted as

$$
\nabla . \mathbf{J}=0
$$

Eq. (14) made the vector as $\mathbf{J}=\left(J_{x}, 0, J_{z}\right)$ i. e. $J_{y}=0$ as the surface is electrically non conducting every where in the fluid flow. Moreover, Maxwell's equations implies that

$$
\nabla \times \mathbf{E}=0
$$

the Eq. (15) resulted as $\mathbf{E}=0$ in the right hand side of Eq. (3) which indicates the drag electron on the ions. The second term $\mathbf{V} \times \mathbf{H}$ of the right hand side of Eq. (3) indicates the contribution of Hall effects. After using Eqs. (14)-(15) in Eq. (3), we arrived at the following results

$$
\begin{gathered}
J_{x}-m_{u} J_{z}=-\sigma H_{y} \widetilde{w} \\
J_{z}+m_{u} J_{x}=\sigma H_{y} \widetilde{u}
\end{gathered}
$$

where $m_{u}=\omega_{e} \tau_{e}$ indicates the Hall parameter and after solving Eqs. (16)-(17) simultaneously, we get

$$
\begin{aligned}
J_{x} & =\frac{\sigma H_{y}}{1+m^{2}}\left(m_{u} \tilde{u}-\tilde{w}\right) \\
J_{z} & =\frac{\sigma H_{y}}{1+m^{2}}\left(\widetilde{u}+m_{u} \widetilde{w}\right)
\end{aligned}
$$

Now the system of boundary layer equations in terms of $\widetilde{x}$ and $\widetilde{z}$ are

$$
\frac{\partial \widetilde{u}}{\partial \widetilde{x}}+\frac{\partial \widetilde{v}}{\partial \widetilde{y}}=0
$$

$$
\begin{gathered}
\frac{\partial \widetilde{u}}{\partial \widetilde{t}}+\widetilde{u} \frac{\partial \widetilde{u}}{\partial \widetilde{y}}+\widetilde{v} \frac{\partial \widetilde{u}}{\partial \widetilde{y}}+\lambda\left[\frac{\partial^{2} \widetilde{u}}{\partial \widetilde{t}^{2}}+2\left(\widetilde{u} \frac{\partial^{2} \widetilde{u}}{\partial \widetilde{x} \partial \widetilde{t}}+\widetilde{v} \frac{\partial^{2} \widetilde{u}}{\partial \widetilde{y} \partial \widetilde{t}}\right)+\widetilde{u}^{2} \frac{\partial^{2} \widetilde{u}}{\partial \widetilde{x}^{2}}+\right. \\
\left.2 \widetilde{u} \widetilde{v} \frac{\partial^{2} \widetilde{u}}{\partial \widetilde{x} \partial \widetilde{y}}+\widetilde{v}^{2} \frac{\partial^{2} \widetilde{u}}{\partial \widetilde{y}^{2}}\right]=\nu \frac{\partial^{2} \widetilde{u}}{\partial \widetilde{y}^{2}}-\frac{1}{\rho}\left(1+\lambda \frac{D}{D t}\right) \frac{J_{z} H_{y}}{t} \quad(21) \\
\frac{\partial \widetilde{w}}{\partial \widetilde{t}}+\widetilde{u} \frac{\partial \widetilde{w}}{\partial \widetilde{y}}+\widetilde{v} \frac{\partial \widetilde{w}}{\partial \widetilde{y}}+\lambda\left[\frac{\partial^{2} \widetilde{w}}{\partial \widetilde{t^{2}}}+2\left(\widetilde{u} \frac{\partial^{2} \widetilde{w}}{\partial \widetilde{x} \partial \widetilde{t}}+\widetilde{v} \frac{\partial^{2} \widetilde{w}}{\partial \widetilde{y} \partial \widetilde{t}}\right)+\right. \\
\left.\widetilde{u}^{2} \frac{\partial^{2} \widetilde{w}}{\partial \widetilde{x}}+2 \widetilde{u} \widetilde{v} \frac{\partial^{2} \widetilde{w}}{\partial \widetilde{x} \partial \widetilde{y}}+\widetilde{v}^{2} \frac{\partial^{2} \widetilde{w}}{\partial \widetilde{y}^{2}}\right]=\nu \frac{\partial^{2} \widetilde{w}}{\partial \widetilde{y}^{2}}+\frac{1}{\rho}\left(1+\lambda \frac{D}{D t}\right) \frac{J_{x} H_{y}}{t}
\end{gathered}
$$

and thermal boundary layer equation is

$$
\frac{\partial \widetilde{T}}{\partial \widetilde{t}}+\widetilde{u} \frac{\partial \widetilde{T}}{\partial \widetilde{x}}+\widetilde{v} \frac{\partial \widetilde{T}}{\partial \widetilde{y}}=\frac{k}{\rho C_{P}} \frac{\partial^{2} \widetilde{T}}{\partial \widetilde{y}^{2}}+\frac{4 \gamma^{2}}{\rho C_{P} t}\left(\widetilde{T}-\widetilde{T}_{\infty}\right)+\frac{Q_{0}^{\prime}}{\rho C_{P} t}\left(\widetilde{T}-\widetilde{T}_{\infty}\right)
$$

with time dependent boundary conditions (Mahdy (2015), Bachok et al. (2012), Palani et al. (2016))

$$
t \leq 0:\left\{\begin{array}{l}
\widetilde{v}(\widetilde{t}, \widetilde{x}, \widetilde{y}, \widetilde{z})=0, \widetilde{u}(\widetilde{t}, \widetilde{x}, \widetilde{y}, \widetilde{z})=0, \widetilde{w}(\widetilde{t}, \widetilde{x}, \widetilde{y}, \widetilde{z})=0 \\
\widetilde{T}(\widetilde{t}, \widetilde{x}, \widetilde{y}, \widetilde{z})=\widetilde{T}_{\infty}, \text { at } \widetilde{y}=0
\end{array}\right.
$$

$$
t>0:\left\{\begin{array}{l}
\widetilde{v}(\widetilde{t}, \widetilde{x}, \widetilde{y}, \widetilde{z})=-\sqrt{\frac{\nu}{\tilde{t}}}, \widetilde{u}(\widetilde{t}, \widetilde{x}, \widetilde{y}, \widetilde{z})=\widetilde{u}_{u}=\frac{\widetilde{x}}{\widetilde{t}}, \widetilde{w}(\widetilde{t}, \widetilde{x}, \widetilde{y}, \widetilde{z})=0, \\
\widetilde{x}, \widetilde{z})=\widetilde{T}_{\infty}+\left(\widetilde{T}_{u}-\widetilde{T}_{\infty}\right) \frac{x}{t^{2}}, \text { at } \widetilde{y}=0 \\
\widetilde{v}(\widetilde{t}, \widetilde{x}, \widetilde{y}, \widetilde{z}) \rightarrow 0, \widetilde{u}(\widetilde{t}, \widetilde{x}, \widetilde{y}, \widetilde{z}) \rightarrow 0, \widetilde{w}(\widetilde{t}, \widetilde{x}, \widetilde{y}, \widetilde{z}) \rightarrow 0 \\
\widetilde{T}(\widetilde{t}, \widetilde{x}, \widetilde{y}, \widetilde{z}) \rightarrow \widetilde{T}_{\infty}, \text { as } \widetilde{y} \rightarrow \infty
\end{array}\right.
$$

The unknowns quantities in Eqs. (20)-(25) promoted as: $\widetilde{t}$ indicates the unsteadiness, $\widetilde{u}$ indicates the axial velocity in $\widetilde{x}$-direction, $\widetilde{w}$ indicates the axial velocity in $\widetilde{z}$-direction, $\rho$ fluid density, $k$ indicates the thermal conductivity, $C_{P}$ indicates the specific heat at constant pressure, $\widetilde{T}$ indicates fluid temperature, $\widetilde{T}_{u}$ indicates the referenced fluid temperature and $\widetilde{T}_{\infty}$ indicates the free stream fluid temperature. Moreover, $U_{u}$ indicates the reference velocity and $L_{u}$ indicates the reference length and by the use of these two quantities, we have the following system

$$
\begin{aligned}
x=\frac{\widetilde{x}}{L_{u}}, y=\frac{\widetilde{y}}{L_{u}} R e^{\frac{1}{2}}, u=\frac{\widetilde{u}}{U_{u}}, v=\frac{\widetilde{v}}{U_{u}} R e^{\frac{1}{2}}, t=\frac{U_{u} \widetilde{t}}{L_{u}}, w=\frac{\widetilde{w}}{U_{u}}, \\
\theta=\frac{\widetilde{T}-\widetilde{T}_{\infty}}{\widetilde{T}_{u}-\widetilde{T}_{\infty}} .
\end{aligned}
$$

Now, the system of Eqs. (21)-(25) will be in dimensionless form after using Eq. (26) as

$$
\frac{\partial u}{\partial x}+\frac{\partial v}{\partial y}=0
$$

$$
\begin{gathered}
\frac{\partial u}{\partial t}+u \frac{\partial u}{\partial y}+v \frac{\partial u}{\partial y}+D e_{u}^{*}\left[\frac{\partial^{2} u}{\partial t^{2}}+2\left(u \frac{\partial^{2} u}{\partial x \partial t}+v \frac{\partial^{2} u}{\partial y \partial t}\right)+u^{2} \frac{\partial^{2} u}{\partial x^{2}}+\right. \\
\left.2 u v \frac{\partial^{2} u}{\partial x \partial y}+v^{2} \frac{\partial^{2} u}{\partial y^{2}}\right]=\frac{\partial^{2} u}{\partial y^{2}}-\frac{M_{u}^{2}}{\left(1+m_{u}^{2}\right) t}(u+m w)-\frac{M_{u}^{2}}{\left(1+m_{u}^{2}\right) t} \\
D e_{u}^{*}\left[\frac{\partial(u+m w)}{\partial t}+v \frac{\partial u}{\partial y}+m u \frac{\partial w}{\partial x}+m v \frac{\partial w}{\partial y}\right]
\end{gathered}
$$

$$
\begin{array}{r}
\frac{\partial w}{\partial t}+u \frac{\partial w}{\partial y}+v \frac{\partial w}{\partial y}+D e_{u}^{*}\left[\frac{\partial^{2} w}{\partial t^{2}}+2\left(u \frac{\partial^{2} w}{\partial x \partial t}+v \frac{\partial^{2} w}{\partial y \partial t}\right)+u^{2} \frac{\partial^{2} w}{\partial x^{2}}+\right. \\
\left.2 u v \frac{\partial^{2} w}{\partial x \partial y}+v^{2} \frac{\partial^{2} w}{\partial y^{2}}\right]=\frac{\partial^{2} w}{\partial y^{2}}+\frac{M_{u}^{2}}{\left(1+m_{u}^{2}\right) t}(m u-w)+\frac{M_{u}^{2}}{\left(1+m_{u}^{2}\right) t} \\
D e_{u}^{*}\left[\frac{\partial(m u-w)}{\partial t}+v m \frac{\partial u}{\partial y}+m u \frac{\partial u}{\partial x}-u \frac{\partial w}{\partial x}-v \frac{\partial w}{\partial y}-m u \frac{\partial w}{\partial x}+w \frac{\partial w}{\partial x}\right]
\end{array}
$$

$$
\frac{\partial \theta}{\partial t}+u \frac{\partial \theta}{\partial x}+v \frac{\partial \theta}{\partial y}=\frac{1}{\operatorname{Pr}} \frac{\partial^{2} \theta}{\partial y^{2}}+\left(\delta_{u}+Q_{u}\right) \frac{\theta}{t}
$$

with

$$
\begin{gathered}
u=u_{t}=\frac{x}{t}, v=v_{u}=-\sqrt{\frac{1}{t}}, \theta=\frac{x}{t^{2}} \text { at } y=0 \\
u=0=w=v, \theta \rightarrow 0, \text { as } y \rightarrow \infty
\end{gathered}
$$

the dimensionless parameters in Eqs. (27)-(32) are

$$
\operatorname{Pr}=\frac{\rho C_{P}}{k}, M_{u}^{2}=\frac{\sigma H_{y}^{2} L_{u}}{\rho U_{u}}, D e_{u}^{*}=\frac{\lambda U_{u}}{L_{u}}, \delta_{u}=\frac{4 \gamma^{2}}{\rho C_{P}}, Q_{u}=\frac{Q_{0}^{\prime}}{\rho C_{P}}
$$

$P r$ indicates the Prandtl number, $M_{u}$ indicates the Hartmann number and $D e_{u}$ indicates the Deborah number.

\section{TWO-PARAMETER LIE SCALING TRANSFORMATIONS}

There are numerous methods to solve the ODEs in literature. But here we used the special class of the Lie scaling group of transformations. The translation method discovered by Sophus Lie and the method was the result of the Invariance of differential equations in the continuous group of symmetries. The applications of this theory are: topology, invariant theory, classical mechanics, relativity, differential geometry, and many more. 


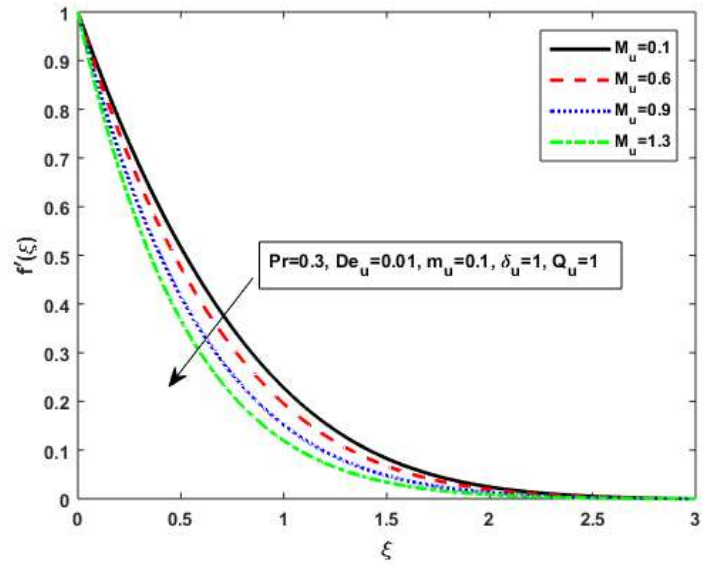

Fig. 2 Impact of $\widetilde{x}$ velocity component for $M_{u}$

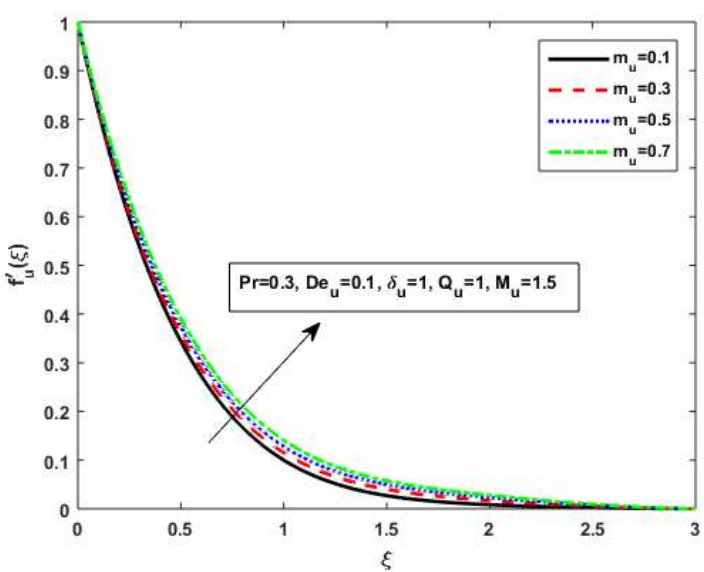

Fig. 3 Impact of $\widetilde{x}$ velocity component for $m_{u}$

Normally, scaling transformations are often referred to as one-parameter transformations ( Mukhopadhyay and Bhattacharyya (2012), Megahed et al. (2003), Ibrahim et al. (2005), Rosmila et al. (2012), Hamad et al. (2012), Reddy (2013), Uddin et al. (2015), Rehman et al. (2018), Uddin et al. (2016b), Uddin et al. (2016a), Rehman et al. (2017), Pal and Roy (2018), Das et al. (2019)) This flow model has three independent variables, so we need to extend the Lie scaling theory from one-parameter to two-parameter Lie scaling transformations. As, three independent variables are transformed to one independent variable. For this model, the two-parameter Lie scaling transformations described as:

$$
\begin{aligned}
\widehat{t}=t C^{\Omega_{1}}, \widehat{x} & =x D^{\Pi_{1}}, \widehat{u}=u C^{\Omega_{2}} D^{\Pi_{2}}, \widehat{v}=v C^{\Omega_{3}} D^{\Pi_{3}} \\
\widehat{y} & =y C^{\Omega_{4}} D^{\Pi_{4}}, \widehat{\theta}=\theta C^{\Omega_{5}} D^{\Pi_{5}}, \widehat{w}=w C^{\Omega_{6}} D^{\Pi_{6}}
\end{aligned}
$$

where $C, D, \Omega_{i}, \Pi_{i}(i=1,2,3,4,5,6)$ are arbitrary constants. The PDEs (27)-(30) according to the boundary conditions (31) and (32) are solved by using two-parameter scaling transformations as Eq. (33). As it is a complex problem and difficult to get the exact solution so we represent the numerical solution after getting the ODEs. Eq. (33) made the system

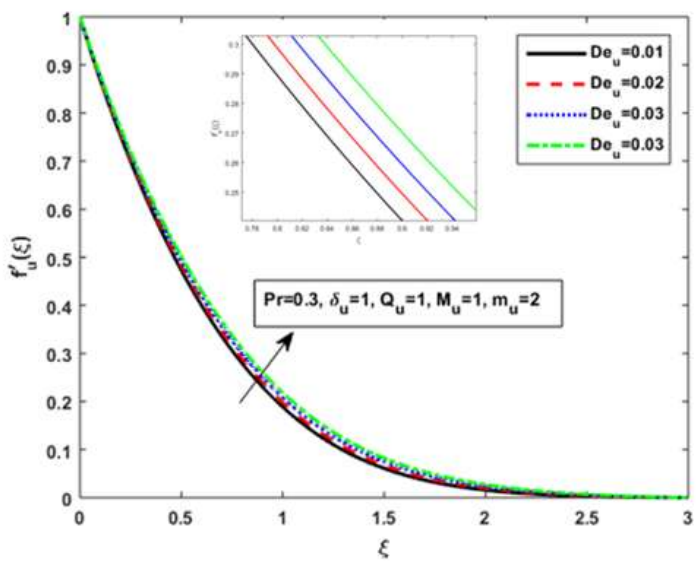

Fig. 4 Impact of $\widetilde{x}$ velocity component for $D e_{u}$

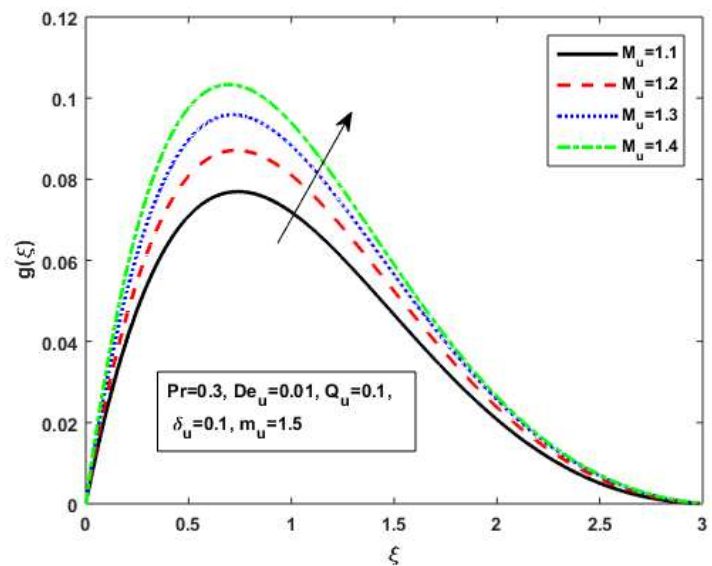

Fig. 5 Impact of $\widetilde{z}$ velocity component for $M_{u}$

of Eqs. (27)-(32) as:

$$
\begin{gathered}
\frac{\partial \widehat{u}}{\partial \widehat{t}} C^{-\Omega_{2}+\Omega_{1}} D^{-\Pi_{2}}+\widehat{u} \frac{\partial \widehat{u}}{\partial \widehat{x}} C^{-2 \Omega_{2}} D^{-2 \Pi_{2}+\Pi_{1}}+\widehat{v} \frac{\partial \widehat{u}}{\partial \widehat{y}} C^{-\Omega_{2}+\Omega_{4}-\Omega_{3}} \\
D^{-\Pi_{4}-\Pi_{3}-\Pi_{2}}+D e_{u}^{*}\left[\frac{\partial^{2} \widehat{u}}{\partial \widehat{t^{2}}} C^{2 \Omega_{1}-\Omega_{2}} D^{-\Pi_{2}}+2\left(\widehat{u} \frac{\partial^{2} \widehat{u}}{\partial \widehat{x} \partial \widehat{t}} C^{\Omega_{1}-2 \Omega_{2}} D^{-\Pi_{1}-2 \Pi_{2}}\right.\right. \\
\left.+\widehat{v} \frac{\partial^{2} \widehat{u}}{\partial \widehat{y} \partial \widehat{t}} C^{-\Omega_{2}+\Omega_{1}-\Omega_{3}+\Omega_{4}} D^{-\Pi_{2}-\Pi_{3}+\Pi_{4}}\right)+\widehat{u}^{2} \frac{\partial^{2} \widehat{u}}{\partial \widehat{x}^{2}} C^{-3 \Omega_{2}} D^{2 \Pi_{1}-3 \Pi_{2}} \\
+2 \widehat{u} \widehat{v} \frac{\partial^{2} \widehat{u}}{\partial \widehat{x} \partial \widehat{y}} C^{-2 \Omega_{2}-\Omega_{3}+\Omega_{4}} D^{-\Pi_{2}+\Pi_{1}+\Pi_{4}-\Pi_{3}}+\widehat{v}^{2} \frac{\partial^{2} \widehat{u}}{\partial \widehat{y}^{2}} C^{-2 \Omega_{3}-\Omega_{2}+2 \Omega_{4}} \\
-\frac{\left.D^{-2 \Pi_{2}+2 \Pi_{4}-\Pi_{2}}\right]=\frac{\partial^{2} \widehat{u}}{\partial \widehat{y}^{2}} C^{2 \Omega_{4}-\Omega_{2}} D^{2 \Pi_{4}-\Pi_{2}}}{\left(1+m_{u}^{2}\right) \widehat{t} C^{-\Omega_{1}}}\left(\widehat{u} C^{-\Omega_{2}} D^{-\Pi_{2}}+m \widehat{w} C^{-\Omega_{6}} D^{-\Pi_{6}}\right) \\
-\frac{M_{u}^{2}}{\left(1+m_{u}^{2}\right) \widehat{t} C^{-\Omega_{1}}} D e_{u}^{*}\left[\frac{\partial \widehat{u}}{\partial \widehat{t}} C^{-\Omega_{2}+\Omega_{1}} D^{-\Pi_{2}}\right. \\
+m \frac{\partial \widehat{w}}{\partial \widehat{t}} C^{-\Omega_{6}+\Omega_{1}} D^{-\Pi_{6}}+\widehat{v} \frac{\partial \widehat{u}}{\partial \widehat{y}} C^{\Omega_{4}-\Omega_{3}-\Omega_{2}} D^{\Pi_{4}-\Pi_{3}-\Pi_{2}} \\
\left.+m \widehat{u} \frac{\partial \widehat{w}}{\partial \widehat{x}} C^{-\Omega_{6}+\Omega_{2}} D^{\Pi_{1}-\Pi_{2}-\Pi_{2}}+m \widehat{v} \frac{\partial \widehat{w}}{\partial \widehat{y}} C^{\Omega_{4}-\Omega_{3}-\Omega_{2}} D^{\Pi_{4}-\Pi_{3}-\Pi_{6}}\right]
\end{gathered}
$$




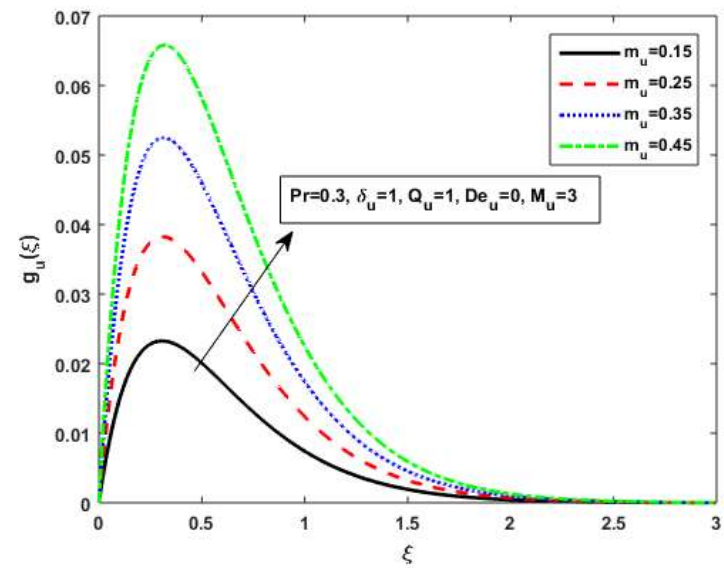

Fig. 6 Impact of $\widetilde{z}$ velocity component for $m_{u}$

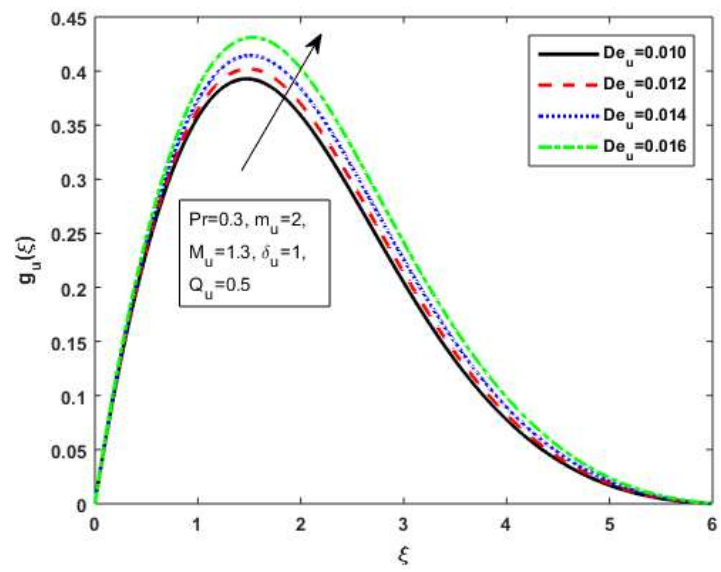

Fig. 7 Impact of $\widetilde{z}$ velocity component for $D e_{u}$

$$
\begin{aligned}
& \frac{\partial \widehat{w}}{\partial \widehat{t}} C^{-\Omega_{6}+\Omega_{1}} D^{-\Pi_{2}}+\widehat{u} \frac{\partial \widehat{w}}{\partial \widehat{x}} C^{-\Omega_{2}+\Omega_{6}} D^{-\Pi_{2}+\Pi_{1}-\Pi_{6}}+\widehat{v} \frac{\partial \widehat{w}}{\partial \widehat{y}} C^{-\Omega_{6}+\Omega_{4}-\Omega_{3}} \\
& \begin{array}{c}
D^{\Pi_{4}-\Pi_{3}-\Pi_{6}}+D e_{u}^{*}\left[\frac{\partial^{2} \widehat{w}}{\partial \widehat{t}^{2}} C^{2 \Omega_{1}-\Omega_{6}} D^{-\Pi_{6}}+2\left(\widehat{u} \frac{\partial^{2} \widehat{w}}{\partial \widehat{x} \partial \widehat{t}} C^{\Omega_{1}-\Omega_{6}-\Omega_{2}} D^{\Pi_{1}}\right.\right. \\
\left.+\widehat{v} \frac{\partial^{2} \widehat{w}}{\partial \widehat{y} \partial \widehat{t}} C^{\Omega_{1}-\Omega_{3}-\Omega_{6}+\Omega_{4}} D^{\Pi_{4}-\Pi_{3}-\Pi_{6}}\right)+\widehat{u}^{2} \frac{\partial^{2} \widehat{w}}{\partial \widehat{x}^{2}} C^{-2 \Omega_{2}-\Omega_{6}}
\end{array} \\
& D^{-2 \Pi_{1}-2 \Pi_{2}-\Pi_{6}}+2 \widehat{u} \widehat{v} \frac{\partial^{2} \widehat{w}}{\partial \widehat{x} \partial \widehat{y}} C^{-\Omega_{2}-\Omega_{3}+\Omega_{4}-\Omega_{6}} D^{\Pi_{1}-\Pi_{3}-\Pi_{2}+\Pi_{4}} \\
& \left.+\widehat{v}^{2} \frac{\partial^{2} \widehat{w}}{\partial \widehat{y}^{2}} C^{-2 \Omega_{3}-\Omega_{6}+2 \Omega_{4}} D^{-2 \Pi_{3}+2 \Pi_{4}-\Pi_{6}}\right]=\frac{\partial^{2} \widehat{w}}{\partial \widehat{y}^{2}} C^{2 \Omega_{4}-\Omega_{6}} D^{2 \Pi_{4}-\Pi_{6}} \\
& +\frac{M_{u}^{2}}{\left(1+m_{u}^{2}\right) \widehat{t} C^{-\Omega_{1}}}\left(m \widehat{u} C^{-\Omega_{2}} D^{-\Pi_{2}}-\widehat{w} C^{-\Omega_{6}} D^{-\Pi_{6}}\right) \\
& +\frac{M_{u}^{2}}{\left(1+m_{u}^{2}\right) \widehat{t} C^{-\Omega_{1}}} D e_{u}^{*}\left[m \frac{\partial \widehat{u}}{\partial \widehat{t}} C^{-\Omega_{2}+\Omega_{1}} D^{-\Pi_{2}}-\frac{\partial \widehat{w}}{\partial \widehat{t}} C^{-\Omega_{6}+\Omega_{1}} D^{-\Pi_{6}}\right. \\
& +m \widehat{u} \frac{\partial \widehat{u}}{\partial \widehat{x}} C^{-2 \Omega_{2}} D^{\Pi_{1}-2 \Pi_{2}}-\widehat{u} \frac{\partial \widehat{w}}{\partial \widehat{x}} C^{-\Omega_{6}-\Omega_{2}} D^{\Pi_{1}-\Pi_{2}-\Pi_{6}}+m \widehat{v} \frac{\partial \widehat{u}}{\partial \widehat{y}} C^{\Omega_{4}-\Omega_{3}-\Omega_{2}} \\
& D^{\Pi_{4}-\Pi_{3}-\Pi_{2}} \widehat{v} \frac{\partial \widehat{w}}{\partial \widehat{y}} C^{-\Omega_{2}+\Omega_{1}} D^{-\Pi_{2}}-m \widehat{u} \frac{\partial \widehat{w}}{\partial \widehat{x}} C^{-\Omega_{6}-\Omega_{2}} D^{\Pi_{1}-\Pi_{2}-\Pi_{6}} \\
& \left.+\widehat{w} \frac{\partial \widehat{w}}{\partial \widehat{x}} C^{-\Omega_{6}} D^{-2 \Pi_{6}+\Pi_{1}}\right]
\end{aligned}
$$

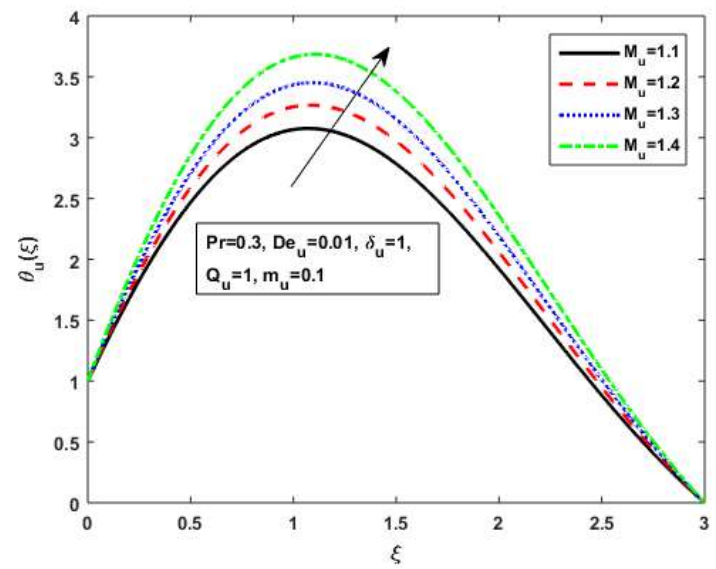

Fig. 8 Impact of heat transfer $\theta(\xi)$ for $M_{u}$

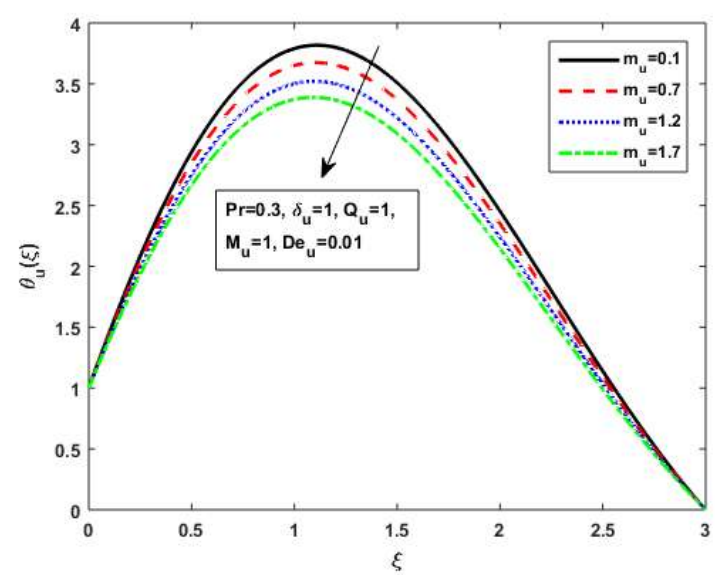

Fig. 9 Impact of heat transfer $\theta(\xi)$ for $m_{u}$

$$
\begin{gathered}
\frac{\partial \widehat{\theta}}{\partial \widehat{t}} C^{\Omega_{1}-\Omega_{5}} D^{-\Pi_{5}}+\widehat{u} \frac{\partial \widehat{\theta}}{\partial \widehat{x}} C^{-\Omega_{2}-\Omega_{5}} D^{\Pi_{2}-\Pi_{2}-\Pi_{5}}+\widehat{v} \frac{\partial \widehat{\theta}}{\partial \widehat{y}} C^{\Omega_{4}-\Omega_{3}-\Omega_{5}} \\
D^{\Pi_{4}-\Pi_{3}-\Pi_{5}}=\frac{1}{P r} \frac{\partial^{2} \widehat{\theta}}{\partial \widehat{y}^{2}} C^{-\Omega_{5}+2 \Omega_{4}} D^{-\Pi_{5}+2 \Pi_{4}}+\left(\delta_{u}+Q_{u}\right) \frac{\widehat{\theta}}{\widehat{t}} C^{\Omega_{1}-\Omega_{5}} D^{-\Pi_{5}}
\end{gathered}
$$

with the following converted boundary conditions

$$
\begin{gathered}
\widehat{u} C^{-\Omega_{2}} D^{-\Pi_{2}}=\frac{\widehat{x}}{\widehat{t}} C^{\Omega_{1}} B^{-\Pi_{1}}, \widehat{v} C^{-\Omega_{3}} D^{-\Pi_{3}}=\sqrt{\frac{C^{\Omega_{1}}}{\widehat{t}}} \\
\widehat{\theta} C^{-\Omega_{5}} D^{-\Pi_{5}}=\frac{\widehat{x}}{\widehat{t}^{2}} C^{-2 \Omega_{1}} D^{-\Pi_{1}}
\end{gathered}
$$

We arrived at the following results

$$
\begin{gathered}
\Omega_{2}=-\Omega_{1}, \Omega_{3}=-\frac{\Omega_{1}}{2}, \Omega_{5}=-2 \Omega_{1} \\
\Pi_{2}=\Pi_{1}, \Pi_{3}=0, \Pi_{5}=\Pi_{1}
\end{gathered}
$$

From Eq. (27)

$$
\frac{\partial \widehat{u}}{\partial \widehat{x}} C^{-\Omega_{2}} D^{-\Pi_{2}+\Pi_{1}}+\frac{\partial \widehat{v}}{\partial \widehat{y}} C^{-\Omega_{3}+\Omega_{4}} D^{-\Pi_{3}+\Pi_{4}}=0
$$




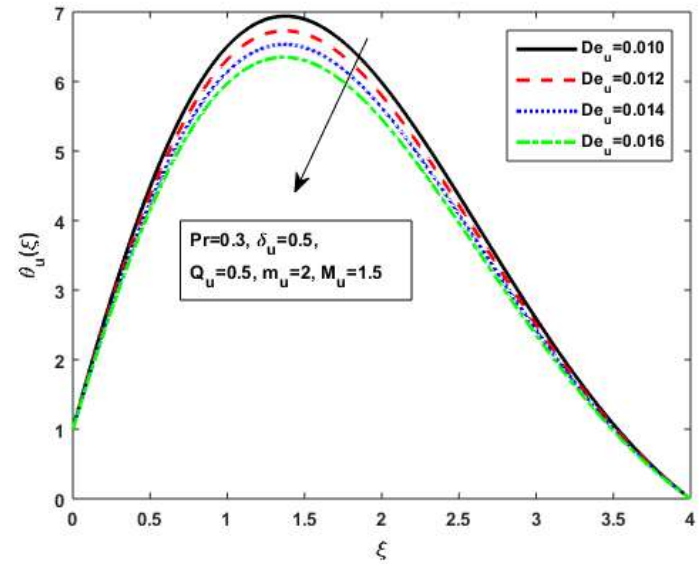

Fig. 10 Impact of heat transfer $\theta(\xi)$ for $D e_{u}$

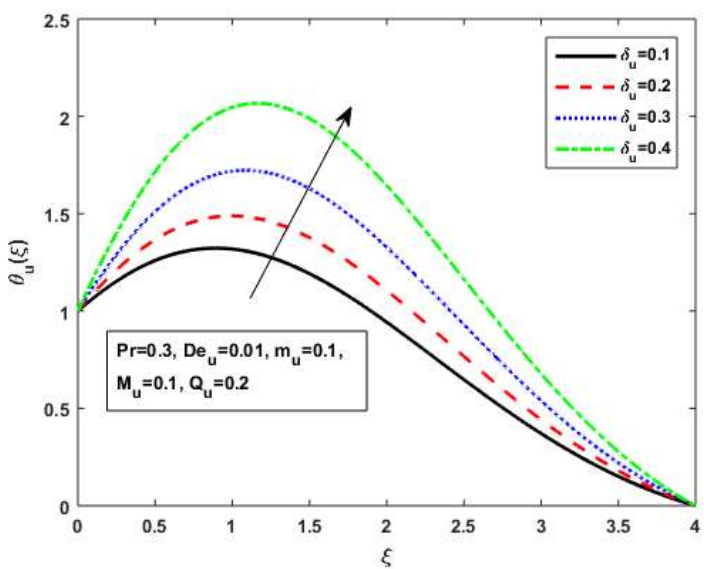

Fig. 11 Impact of heat transfer $\theta(\xi)$ for $\delta_{u}$

On comparing

$$
\Omega_{4}=\frac{\Omega_{1}}{2}, \Pi_{4}=0, \Omega_{6}=-\Omega_{1}, \Pi_{6}=\Pi_{1}
$$

Eq. (33) resulted as after using Eqs. (39)-(40) and (42)

$$
\begin{aligned}
\widehat{t}=t C^{\Omega_{1}}, \widehat{x} & =x D^{\Pi_{1}}, \widehat{u}=u C^{-\Omega_{1}} D^{\Pi_{1}}, \widehat{v}=v C^{-\frac{\Omega_{1}}{2}}, \\
\widehat{y} & =y C^{\frac{\Omega_{1}}{2}}, \widehat{\theta}=\theta C^{-2 \Omega_{1}} D^{\Pi_{1}}, \widehat{w}=w C^{-\Omega_{1}} D^{\Pi_{1}}
\end{aligned}
$$

\subsection{Absolute Invariants}

Eqs. (33) and (43) promoted to the following result:

$$
\frac{\widehat{y}}{\widehat{t}^{\frac{1}{2}}}=\frac{y}{t^{\frac{1}{2}}}
$$

with the help of Eq. (44), the first absolute invariant is

$$
\xi=\widehat{y} \widehat{t}^{\frac{-1}{2}}
$$

and the remaining absolute invariants are:

$$
\frac{d f_{u}(\xi)}{d \xi}=\frac{\widehat{u}}{\widehat{x}} \widehat{t}, h_{u}(\xi)=\frac{\widehat{v}}{\widehat{t}^{\frac{1}{2}}}, g_{u}(\xi)=\frac{\widehat{w}}{\widehat{x} \widehat{t}^{-1}}, \theta(\xi)=\frac{\widehat{\theta}}{\widehat{t}^{-2} \widehat{x}}
$$

where $\xi$ is the similarity independent variable.

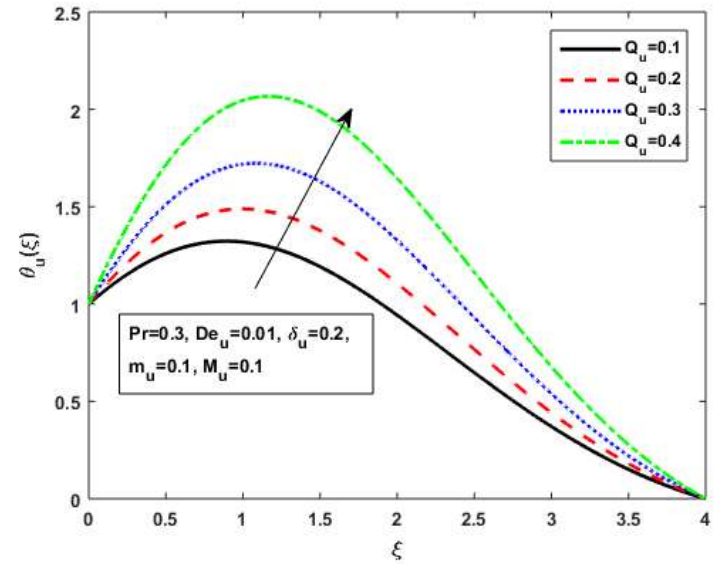

Fig. 12 Impact of heat transfer $\theta(\xi)$ for $Q_{u}$

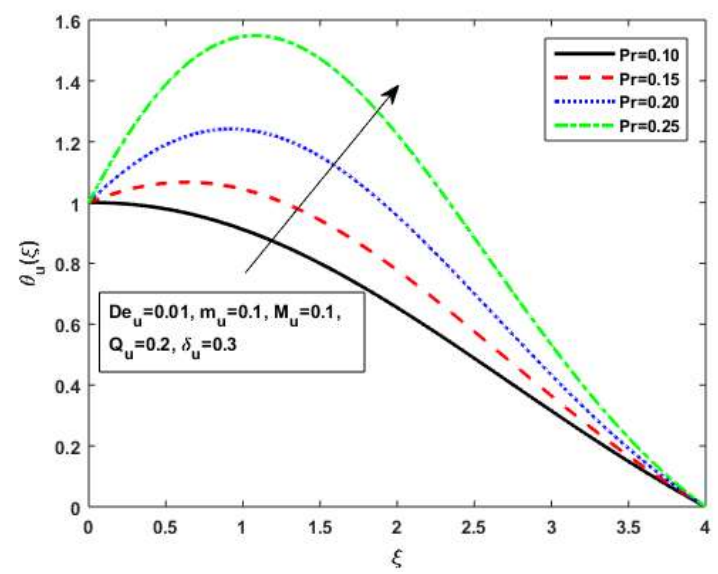

Fig. 13 Impact of heat transfer $\theta(\xi)$ for $P r$

\subsection{Reduced governing system}

Introducing the stream function $\psi(x, y, t)$ as

$$
u=\frac{\partial \psi}{\partial y}, v=-\frac{\partial \psi}{\partial x}
$$

Eqs. (45)-(47), we arrived at the value of $\psi(x, y, t)$

$$
\psi=-x t^{\frac{-1}{2}} h_{u}(\xi)
$$

where we get the value of $h_{u}$

$$
\psi=x t^{\frac{-1}{2}}\left(f_{u}(\xi)-\xi \frac{d f_{u}(\xi)}{d \xi}\right)
$$

after comparing, the system of Eqs. (34)-(35) in terms of absolute invariants Eq. (46)

$$
h_{u}(\xi)=-\left(f_{u}(\xi)-\xi \frac{d f_{u}(\xi)}{d \xi}\right)
$$


The system of Eqs. (34)-(38) in terms of absolute invariants Eq. (46)

$$
\begin{aligned}
& -\frac{d f_{u}(\xi)}{d \xi}-\frac{1}{2} \xi \frac{d^{2} f_{u}(\xi)}{d \xi^{2}}+\left(\frac{d f_{u}(\xi)}{d \xi}\right)^{2}+h_{u}(\xi) \frac{d^{2} f_{u}(\xi)}{d \xi^{2}} \\
& =\frac{d^{3} f_{u}(\xi)}{d \xi^{3}}-D e_{u}\left[2 \frac{d f_{u}(\xi)}{d \xi}-\frac{1}{2} \frac{d^{2} f_{u}(\xi)}{d \xi^{2}}+\xi \frac{d^{2} f_{u}(\xi)}{d \xi^{2}}+\frac{1}{4} \xi^{2} \frac{d^{3} f_{u}(\xi)}{d \xi^{3}}\right. \\
& +2\left(-\frac{d f_{u}(\xi)^{2}}{d \xi}-\frac{1}{2} \frac{d^{2} f_{u}(\xi)}{d \xi^{2}}-\frac{3}{2} h_{u}(\xi) \frac{d^{2} f_{u}(\xi)}{d \xi^{2}}-\frac{1}{2} \xi \frac{d^{3} f_{u}(\xi)}{d \xi^{3}} h_{u}(\xi)\right) \\
& \left.+2 h_{u}(\xi) \frac{d f_{u}(\xi)}{d \xi}+h_{u}^{2} \frac{d^{3} f_{u}(\xi)}{d \xi^{3}}\right]-\frac{M_{u}^{2}}{1+m_{u}^{2}}\left(\frac{d f_{u}(\xi)}{d \xi}+m g_{u}(\xi)\right) \\
& -\frac{M_{u}^{2}}{1+m_{u}^{2}} D e_{u}\left[-\frac{d f_{u}(\xi)}{d \xi}+\frac{d^{2} f_{u}(\xi)}{d \xi^{2}}+m\left(-g_{u}(\xi)+\xi \frac{d g_{u}(\xi)}{d \xi}\right)+\right. \\
& \left.h_{u}(\xi) \frac{d^{2} f_{u}(\xi)}{d \xi^{2}}+m g_{u}(\xi) \frac{d f_{u}(\xi)}{d \xi}+m h_{u}(\xi) \frac{d g_{u}(\xi)}{d \xi}\right] \\
& -g_{u}(\xi)-\frac{1}{2} \xi \frac{d g_{u}(\xi)}{d \xi}+g_{u}(\xi) \frac{d f_{u}(\xi)}{d \xi}=\frac{d^{2} g_{u}(\xi)}{d \xi^{2}}-D e_{u}\left[2 g_{u}(\xi)\right. \\
& +\frac{7}{4} \xi \frac{d g_{u}(\xi)}{d \xi}+\frac{1}{4} \xi^{2} \frac{d^{2} g_{u}(\xi)}{d \xi^{2}}+2\left(-\frac{d f_{u}(\xi)}{d \xi} g_{u}(\xi)-\frac{1}{2} \xi \frac{d g_{u}(\xi)}{d \xi}+\frac{3}{2} h_{u}(\xi)\right. \\
& \left.\left.-\frac{1}{2} \xi \frac{d^{2} g_{u}(\xi)}{d \xi^{2}}\right)+2 h_{u}(\xi) \frac{d f_{u}(\xi)}{d \xi} \frac{d g_{u}(\xi)}{d \xi}+h_{u}^{2}(\xi) \frac{d^{2} g_{u}(\xi)}{d \xi^{2}}\right]+\frac{M_{u}^{2}}{1+m_{u}^{2}} \\
& \left(m_{u} \frac{d f_{u}(\xi)}{d \xi}-g_{u}(\xi)\right)+\frac{M_{u}^{2}}{1+m_{u}^{2}} D e_{u}\left[-m_{u} \frac{d f_{u}(\xi)}{d \xi}-\frac{1}{2} m_{u} \xi \frac{d^{2} f_{u}(\xi)}{d \xi^{2}}\right. \\
& +g_{u}(\xi)-\frac{1}{2} \xi \frac{d g_{u}(\xi)}{d \xi}+m_{u}\left(\frac{d f_{u}(\xi)}{d \xi}\right)^{2}-\frac{d f_{u}(\xi)}{d \xi} g_{u}(\xi)+m_{u} h_{u}(\xi) \frac{d^{2} f_{u}(\xi)}{d \xi^{2}} \\
& \left.+h_{u}(\xi) g_{u}(\xi)+g_{u}^{2}(\xi)\right]
\end{aligned}
$$

where $D e_{u}=\frac{D e_{u}^{*}}{t}$

$$
\begin{array}{r}
-2 \theta_{u}(\xi)-\frac{1}{2} \frac{d \theta_{u}(\xi)}{d \xi}+\theta_{u}(\xi) \frac{d f_{u}(\xi)}{d \xi}+h_{u}(\xi) \frac{d \theta_{u}(\xi)}{d \xi}=\frac{1}{\operatorname{Pr}} \frac{d^{2} \theta_{u}(\xi)}{d \xi^{2}}+ \\
\left(\delta_{u}+Q_{u}\right) \theta_{u}(\xi)
\end{array}
$$

with boundary conditions in terms of absolute invariants

$$
\begin{gathered}
f_{u}(\xi)=1, \frac{d f_{u}(\xi)}{d \xi}=1, g_{u}(\xi)=0, \theta_{u}(\xi)=1, \text { at } \xi=0 \\
f_{u}(\xi)=0, g_{u}(\xi)=0, \theta_{u}(\xi)=0 \text { as } \xi \rightarrow \infty
\end{gathered}
$$

\subsection{Verification}

To verify the result, Eq. (51) reduce to Mukhopadhyay and Bhattacharyya (2012), if we choose $M=1$ and $\beta=0$ after setting $D e_{u}=M_{u}=0$ and $(h(\xi) \rightarrow f(\xi), t \rightarrow \infty)$. This approached to the validation of the system which has been solved by two-parameter scaling transformations.

\section{ANALYSIS}

The ODEs (51)-(53) along with the unsteady boundary conditions (54)(55) are solved numerically by bvp4c in MATLAB. The numerical solution performed with the following criteria: $0.1 \leq M_{u} \leq 1,0.1 \leq m_{u} \leq$ $1,1 \leq R_{u} \leq 4,0.01 \leq D e_{u} \leq 0.1,0.1 \leq \operatorname{Pr} \leq 0.4,0.1 \leq Q_{u} \leq$ $1.5,0.1 \leq \delta_{u} \leq 1.5$. Figures $2-13$ are presented for the pertinent parameters like Deborah number $D e_{u}$, Hartmann number $M_{u}$, Hall effects parameter $m_{u}$, Prandtl number $\operatorname{Pr}$, thermal radiation parameter $d_{u}$ and heat generation-absorption $Q_{u}$. For the Hartmann number $M_{u}$, Figures 2, 5 and 8 are plotted for the primary flow $f^{\prime}(\xi)$, secondary flow $g(\xi)$

and transport of internal energy . It is the fact that the Lorentz force reduced the primary flow, while the secondary flow trend can be seen in the reverse. The Lorentz force increases the temperature of the particles and the transport of heat increases when the Hartmann number increases. For the Hall parameter $m_{u}$, Figures 3, 6 and 9 are plotted. The Hall parameter increased the primary flow at the center of the channel, but there is no flow at the end of the channel. Secondary flow parabolically resulted in Hall parameter and increased behavior throughout the channel. Physically, the increase in the Hall parameter resulted in a decrease in the capacitance of the fluid flow components. However, the transport of internal energy decreased due to the Hall effects. For the Deborah number $D e_{u}$, Figures 4, 7 and 10 are presented. Maxwell's fluid properties increased the primary and secondary flow, but the reversing behavior is seen through the channel in the case of internal energy transport. There is the value of the maximum $D e_{u}=0.1$, which is the right value for showing non-Newtonian fluid effects for this model since it behaved like a viscous fluid for the low Deborah level. Thermal radiation graphed in Figure 11. The transfer of internal energy in the channel was increased due to thermal radiation. The heat generation-absorption and the Prandtl number are listed in Figures 12-13. It can be observed that the internal energy transfer is increased due to heat generation-absorption and Prandtl.

\section{CONCLUSIONS}

The present research is the contribution of the Hall effects to the timedependent UCM. This fluid flow has passed through the infinite length of the stretching layer with thermal radiation and heat generation-absorption influences. The governing system of equations is converted from PDEs to ODEs with the help of two-parameter transformations. Then solved by the MATLAB bvp4c and presented via the graphs. The following results were described in the graphs as:

1. Primary velocity $f_{u}^{\prime}(\xi)$ having decreased behavior with magnetic field parameter $M_{u}$ increasing. Secondary velocity $g_{u}(\xi)$ parabolically increased not only at the center of the wall, but also away from the center when an increase occurred in $M_{u}$. The same behavior can be observed for the temperature field $\theta_{u}(\xi)$.

2. Hall parameter $m_{u}$ raised the primary velocity $f_{u}^{\prime}(\xi)$ at the center of the channel, but there is no result on the boundary wall. Hall parameter $m_{u}$ increased the secondary velocity $g_{u}(\xi)$ not only at the center of the channel, but also at the boundary of the wall. Hall parameter $m_{u}$ reduced the transport of internal energy $\theta_{u}(\xi)$ through the channel.

3. Primary velocity $f_{u}^{\prime}(\xi)$ increased at the center of the channel when the Deborah number $D e_{u}$ increased. Secondary velocity $g_{u}(\xi)$ parabolically increased at the center of the channel, but there is no result at the boundary wall when the Deborah number $D e_{u}$ is increased. Deborah number $D e_{u}$ decreased the transport of internal energy $\theta_{u}(\xi)$.

4. Thermal radiation and heat generation-absorption parameters increased the transport of the internal energy $\theta_{u}(\xi)$ of the fluid model.

\section{ACKNOWLEDGEMENTS}

To the FHMT Editorial Board, I am very grateful. This work can not be published successfully without their encouragement and effort. I am also grateful to the reviewers who have done a very good job.

\section{NOMENCLATURE}

$\mathbf{A}_{1}$

$a_{u}$

C

$C_{p}$

De

$\mathbf{E}$

$H_{y}$

$\mathbf{J}$
Rivlin-Eriksen tensor material acceleration $\left(\frac{m}{s . s}\right)$ heat capacity $\left(\mathrm{J} / \mathrm{m}^{3} \cdot \mathrm{K}\right)$ specific heat $(\mathrm{J} / \mathrm{kg} \cdot \mathrm{K})$ Deborah number electric field vector uniform magnetic field strength current density vector 


$\begin{array}{ll}k & \text { thermal conductivity }(\mathrm{W} / \mathrm{m} \cdot \mathrm{K}) \\ L_{u} & \text { reference length }(m) \\ M_{u} & \text { Hartmann number } \\ m_{u} & \text { Hall parameter } \\ P r & \text { Prandtl number } \\ Q_{u} & \text { heat generation-absorption parmeter } \\ \mathbf{S} & \text { extra stress tensor } \\ t & \text { time }(s) \\ T & \text { temperature }(K) \\ u & \text { interfacial velocity }(\mathrm{m} / \mathrm{s}) \\ x & \text { coordinate }(m) \\ \text { Greek Symbols } \\ \delta & \text { thermal radiation parameter } \\ \varepsilon & \text { total emissivity } \\ \rho & \text { density }\left(\mathrm{kg} / \mathrm{m}^{3}\right) \\ \sigma & \text { Stefan-Boltzmann constant }\left(\mathrm{W} / \mathrm{m}^{2} \cdot \mathrm{K}^{4}\right) \\ \text { Subscripts } & \\ u & \text { reference enviroment } \\ e & \text { electron } \\ \infty & \text { ambient enviroment }\end{array}$

\section{REFERENCES}

Abbas, Z., Wang, Y., Hayat, T., and Oberlack, M., 2010, "Mixed convection in the stagnation-point flow of a Maxwell fluid towards a vertical stretching surface," Nonlinear Analysis: Real World Applications, 11(4), 3218-3228, doi:https://doi.org/10.1016/j.nonrwa.2009.11.016.

Abel, M.S., Tawade, J.V., and Nandeppanavar, M.M., 2012, "MHD flow and heat transfer for the upper-convected Maxwell fluid over a stretching sheet," Meccanica, 47(2), 385-393, doi:https://doi.org/10.1007/s11012011-9448-7.

Alkasasbeh, H., Swalmeh, M., Bani Saeed, H., Al Faqih, F., and Talafha, A., 2020, "Investigation on cnts-water and human blood based casson nanofluid flow over a stretching sheet under impact of magnetic field," Frontiers in Heat and Mass Transfer, 14, doi:10.5098/hmt.14.15.

Awais, M., Hayat, T., Alsaedi, A., and Asghar, S., 2014, "Time-dependent three-dimensional boundary layer flow of a Maxwell fluid," Computers \& Fluids, 91, 21-27, doi:https://doi.org/10.1016/j.compfluid.2013.12.002.

Bachok, N., Ishak, A., and Pop, I., 2012, "Unsteady boundary-layer flow and heat transfer of a nanofluid over a permeable stretching/shrinking sheet," International Journal of Heat and Mass Transfer, 55(7-8), 21022109, doi:https://doi.org/10.1016/j.ijheatmasstransfer.2011.12.013.

Bilal, M., Sagheer, M., and Hussain, S., 2018, "Three dimensional MHD upper-convected Maxwell nanofluid flow with nonlinear radiative heat flux," Alexandria Engineering Journal, 57(3), 1917-1925, doi:https://doi.org/10.1016/j.aej.2017.03.039.

Das, K., Chakraborty, T., and Kundu, P.K., 2019, "Lie Group Transformation for Double-Diffusive Free Convection Nanofluid Flow Over an Inclined Plane," Proceedings of the National Academy of Sciences, India Section A: Physical Sciences, 89(2), 387-396, doi:https://doi.org/10.1007/s40010-017-0472-4.

Dharmaiah, G., Baby Rani, C., Vedavathi, N., and Balamurugan, K., 2020, "Hall and ion slip effects on Ag-water based MHD nanofluid flow over a semi-infinite vertical plate embedded in a porous medium," Frontiers in Heat and Mass Transfer, 14, doi:10.5098/hmt.14.6.

Farooq, U., Lu, D., Munir, S., Ramzan, M., Suleman, M., and Hussain, S., 2019, "MHD flow of Maxwell fluid with nanomaterials due to an exponentially stretching surface," Scientific Reports, 9(1), 1-11, doi:https://doi.org/10.1038/s41598-019-43549-0.
Gireesha, B., Mahanthesh, B., Gorla, R.S.R., and Krupalakshmi, K., 2018, "Mixed convection two-phase flow of Maxwell fluid under the influence of non-linear thermal radiation, non-uniform heat source/sink and fluid-particle suspension," Ain Shams Engineering Journal, 9(4), 735746, doi:https://doi.org/10.1016/j.asej.2016.04.020.

Hall, E.H., et al., 1879, "On a new action of the magnet on electric currents," American Journal of Mathematics, 2(3), 287-292, doi: $10.2307 / 2369245$.

Hamad, M., Uddin, M.J., and Ismail, A.M., 2012, "Radiation effects on heat and mass transfer in MHD stagnation-point flow over a permeable flat plate with thermal convective surface boundary condition, temperature dependent viscosity and thermal conductivity," Nuclear Engineering and Design, 242, 194-200, doi:https://doi.org/10.1016/j.nucengdes.2011.09.005.

Harris, J., 1977, Rheology and non-Newtonian flow, Longman Publishing Group. URL https://link.springer.com/book/10.1007/ 978-3-319-01053-3.

Hayat, T., Iqbal, Z., Mustafa, M., and Alsaedi, A., 2012, "Momentum and heat transfer of an upper-convected Maxwell fluid over a moving surface with convective boundary conditions," Nuclear Engineering and Design, 252, 242-247, doi:DOI: 10.1016/j.nucengdes.2012.07.012.

Huang, C.J., Hsu, H.P., and Ay, H.c., 2019, "Influence of mhd on free convection of non-newtonian fluids over a vertical permeable plate in porous media with internal heat generation," Frontiers in Heat and Mass Transfer, 13, doi:10.6125/15-0316-844.

Hussain, T., Hussain, S., and Hayat, T., 2016, "Impact of double stratification and magnetic field in mixed convective radiative flow of Maxwell nanofluid," Journal of Molecular Liquids, 220, 870-878, doi:https://doi.org/10.1016/j.molliq.2016.05.012.

Ibrahim, F., Mansour, M., Hamad, M.A., et al., 2005, "Lie-group analysis of radiative and magnetic field effects on free convection and mass transfer flow past a semi-infinite vertical flat plate," Electronic Journal of Differential Equations, 2005, 1-17. URL http://ejde.math. txstate.edu.

Jamil, M., and Fetecau, C., 2010, "Helical flows of Maxwell fluid between coaxial cylinders with given shear stresses on the boundary," Nonlinear Analysis: Real World Applications, 11(5), 4302-4311, doi:https://doi.org/10.1016/j.nonrwa.2010.05.016.

Jana, R.N., and Datta, N., 1977, "Hall effects on unsteady Couette flow," International Journal of Engineering Science, 15(1), 35-43, doi:https://doi.org/10.1016/0020-7225(77)90067-2.

Kaprawi, S., Astuti, H., and Sri, P., 2019, "Natural convection of nanofluids past an accelerated vertical plate with variable wall temperature by presence of the radiation," Frontiers in Heat and Mass Transfer, 13, doi:10.5098/hmt.13.3.

Kashyap, K.P., Ojjela, O., and Das, S.K., 2019, "MHD slip flow of chemically reacting UCM fluid through a dilating channel with heat source/sink," Nonlinear Engineering, 8(1), 523-533, doi:https://doi.org/10.1515/nleng-2018-0036.

Khoshrouye Ghiasi, E., and Saleh, R., 2018, "2D flow of Casson fluid with non-uniform heat source/sink and Joule heating," Frontiers in Heat and Mass Transfer, 12, doi:10.5098/hmt.12.4.

Krishna, M.V., and Jyothi, K., 2018, "Hall effects on MHD rotating flow of a visco-elastic fluid through a porous medium over an infinite oscillating porous plate with heat source and 
chemical reaction," Materials Today: Proceedings, 5(1), 367-380, doi:https://doi.org/10.1016/j.matpr.2017.11.094.

Mahanthesh, B., Gireesha, B., and Raju, C., 2017, "CattaneoChristov heat flux on UCM nanofluid flow across a melting surface with double stratification and exponential space dependent internal heat source," Informatics in Medicine Unlocked, 9, 26-34, doi:https://doi.org/10.1016/j.imu.2017.05.008.

Mahdy, A., 2015, "Heat transfer and flow of a Casson fluid due to a stretching cylinder with the Soret and Dufour effects," Journal of Engineering Physics and Thermophysics, 88(4), 928-936, doi:https://doi.org/10.1007/s10891-015-1267-6.

Mandal, G., and K. Mandal, K., 1983, "Effect of Hall current on MHD Couette flow between thick arbitrarily conducting plate in a rotating system," Journal of the Physical Society of Japan, 52(2), 470-477, doi:https://doi.org/10.1143/JPSJ.52.470.

Maripala, S., and Naikoti, K., 2015, "Hall effects on unsteady MHD free convection flow over a stretching sheet with variable viscosity and viscous dissipation," World Applied Sciences Journal, 33(6), 1032-1041, doi:10.5829/idosi.wasj.2015.33.06.263.

Megahed, A.A., Komy, S.R., and Afify, A.A., 2003, "Similarity analysis in magnetohydrodynamics: Hall effects on free convection flow and mass transfer past a semi-infinite vertical flat plate," International Journal of Non-Linear Mechanics, 38(4), 513-520, doi:https://doi.org/10.1016/S0020-7462(01)00077-4.

Mukhopadhyay, S., and Bhattacharyya, K., 2012, "Unsteady flow of a Maxwell fluid over a stretching surface in presence of chemical reaction," Journal of the Egyptian Mathematical Society, 20(3), 229-234, doi:http://dx.doi.org/10.1016/j.joems.2012.08.019.

Nagy, T., and Demendy, Z., 1995, "Effects of Hall currents and Coriolis force on Hartmann flow under general wall conditions," Acta Mechanica, 113(1-4), 77-91, doi:https://doi.org/10.1007/BF01212635.

Ndlovu, P., and Moitsheki, R., 2018, "Thermal analysis of natural convection and radiation heat transfer in moving porous fins," Frontiers in Heat and Mass Transfer, 12, doi:10.5098/hmt.12.7.

Oosthuizen, P., and Manna, R., 2019, "Numerical and experimental investigations of natural convective heat transfer from two-sided diagonally inclined square plates having a finite thickness," Frontiers in Heat and Mass Transfer, 13, doi:10.5098/hmt.13.7.

Padma, G., and Suneetha, S., 2018, "Hall effects on MHD Flow through Porous Medium in a Rotating Parallel Plate Channel," International Journal of Applied Engineering Research, 13(11), 97729789. URL https://www.ripublication.com/ijaer18/ ijaerv13n11_143.pdf.

Pal, D., and Roy, N., 2018, "Lie group transformation on MHD doublediffusion convection of a Casson nanofluid over a vertical stretching/shrinking surface with thermal radiation and chemical reaction," International Journal of Applied and Computational Mathematics, 4(1), 13, doi:https://doi.org/10.1007/s40819-017-0449-7.

Palani, S., Kumar, B.R., and Kameswaran, P.K., 2016, "Unsteady MHD flow of an UCM fluid over a stretching surface with higher order chemical reaction," Ain Shams Engineering Journal, 7(1), 399-408, doi:https://doi.org/10.1016/j.asej.2015.11.021.

Rajagopal, K., and Gupta, A., 1984, "An exact solution for the flow of a non-Newtonian fluid past an infinite porous plate," Meccanica, 19(2), 158-160, doi:https://doi.org/10.1007/BF01560464.
Raju, M.C., Reddy, N.A., and Varma, S.V.K., 2011, "Hall-current effects on unsteady magnetohydrodynamics flow between stretching sheet and an oscillating porous upper parallel plate with constant suction," Thermal Science, 15(2), 527-536, doi:10.2298/TSCI091120050C.

Reddy, B.S.K., Krishna, M.V., Rao, K.S.N., and Vijaya, R.B., 2018, "HAM Solutions on MHD flow of Nano-fluid through Saturated Porous medium with Hall effects," doi:10.1016/j.matpr.2017.11.062.

Reddy, M.G., 2013, "Lie group analysis of heat and mass transfer effects on steady mhd free convection flow past an inclined surface with viscous dissipation," URL www. jafmonline. net.

Rehman, K.U., Malik, A.A., Malik, M., and Hayat, T., 2017, "Generalized Lie symmetry analysis for non-linear differential equations: A purely viscous fluid model," Results in physics, 7, 3537-3542, doi:https://doi.org/10.1016/j.rinp.2017.09.020.

Rehman, K.U., Malik, A.A., Malik, M., Tahir, M., and Zehra, I., 2018, "On new scaling group of transformation for Prandtl-Eyring fluid model with both heat and mass transfer," Results in Physics, 8, 552-558, doi:https://doi.org/10.1016/j.rinp.2017.12.071.

Rosmila, A., Kandasamy, R., and Muhaimin, I., 2012, "Lie symmetry group transformation for MHD natural convection flow of nanofluid over linearly porous stretching sheet in presence of thermal stratification," Applied Mathematics and Mechanics, 33(5), 593-604, doi:https://doi.org/10.1007/s10483-012-1573-9.

Sadeghy, K., Najafi, A.H., and Saffaripour, M., 2005, "Sakiadis flow of an upper-convected Maxwell fluid," International Journal of Non-Linear Mechanics, 40(9), 1220-1228, doi:https://doi.org/10.1016/j.ijnonlinmec.2005.05.006.

Sato, H., 1961, "The Hall effect in the viscous flow of ionized gas between parallel plates under transverse magnetic field," Journal of the Physical Society of Japan, 16(7), 1427-1433, doi:https://doi.org/10.1143/JPSJ.16.1427.

Seth, G., Kumbhakar, B., and Sharma, R., 2016, "Unsteady MHD free convection flow with Hall effect of a radiating and heat absorbing fluid past a moving vertical plate with variable ramped temperature," Journal of the Egyptian Mathematical Society, 24(3), 471-478, doi:https://doi.org/10.1016/j.joems.2015.07.007.

Sreedevi, G., Rao, R.R., Rao, D.P., and Chamkha, A., 2016, "Combined influence of radiation absorption and Hall current effects on MHD double-diffusive free convective flow past a stretching sheet," Ain Shams Engineering Journal, 7(1), 383-397, doi:https://doi.org/10.1016/j.asej.2015.11.024.

Srinivasacharya, D., and Jagadeeshwar, P., 2017, "MHD flow with Hall current and Joule heating effects over an exponentially stretching sheet," Nonlinear Engineering, 6(2), 101-114, doi:https://doi.org/10.1515/nleng-2016-0035.

Tie-Gang, F., Ji, Z., and Shan-Shan, Y., 2009, "Viscous flow over an unsteady shrinking sheet with mass transfer," Chinese Physics Letters, 26(1), 014703. URL https://iopscience.iop.org/ article/10.1088/0256-307x/26/1/014703.

Tufail, M.N., Saleem, M., and Chaudhry, Q.A., 2020, "An oscillation effect on MHD radiative Casson fluid flows in an asymmetric channel through group theoretical analysis," Canadian Journal of Physics, 98(1), 81-88, doi:10.1139/cjp-2018-0723. 
Uddin, M., Bég, O.A., Amran, N., and Ismail, A.M., 2015, "Lie group analysis and numerical solutions for magneto-convective slip flow of nanofluid over a moving plate with Newtonian heating boundary condition," Canadian Journal of Physics, 93(12), 1501-1509, doi:10.1139/cjp2014-0601.

Uddin, M., Khan, W., and Ismail, A.M., 2016a, "Two parameter scaling group for unsteady convective magnetohydrodynamic flow," Alexandria Engineering Journal, 55(2), 829-835, doi:https://doi.org/10.1016/j.aej.2016.02.004.

Uddin, M., Rashidi, M., Alsulami, H.H., Abbasbandy, S., and Freidoonimeh, N., 2016b, "Two parameters Lie group analysis and numerical solution of unsteady free convective flow of nonNewtonian fluid," Alexandria Engineering Journal, 55(3), 2299-2308, doi:https://doi.org/10.1016/j.aej.2016.05.009.

Veera Krishna, M., and Jyothi, K., 2018, "Hall effects on MHD Rotating flow of a Visco-elastic Fluid through a Porous medium Over an Infinite Oscillating Porous Plate with Heat source and Chemical reaction," Materials Today: Proceedings, 5(1, Part 1), 367 380, doi:https://doi.org/10.1016/j.matpr.2017.11.094. International Conference on Processing of Materials, Minerals and Energy (July 29th Ü 30th) 2016, Ongole, Andhra Pradesh, India.

VeeraKrishna, M., Subba Reddy, G., and Chamkha, A., 2018, "Hall ef- fects on unsteady MHD oscillatory free convective flow of second grade fluid through porous medium between two vertical plates," Physics of Fluids, 30(2), 023106, doi:https://doi.org/10.1063/1.5010863.

Vijaya, K., and Ramana Reddy, G., 2020, "Influence of critical parameters of thermophorosis on MHD non-Darcy flow of a Casson fluid past a permeable stretching sheet," Frontiers in Heat and Mass Transfer, 14, doi:10.5098/hmt.14.12.

Zhao, J., Zheng, L., Zhang, X., and Liu, F., 2016, "Unsteady natural convection boundary layer heat transfer of fractional Maxwell viscoelastic fluid over a vertical plate," International Journal of Heat and Mass Transfer, 97, 760-766, doi:https://doi.org/10.1016/j.ijheatmasstransfer.2016.02.059.

Zheng, L., Li, C., Zhang, X., and Gao, Y., 2011, "Exact solutions for the unsteady rotating flows of a generalized Maxwell fluid with oscillating pressure gradient between coaxial cylinders," Computers \& Mathematics with Applications, 62(3), 1105-1115, doi:https://doi.org/10.1016/j.camwa.2011.02.044.

Zhou, Z., Wang, Z., and Yang, M., 2020, "Double diffusive natural convection in open cavity under the Soret and Dufour effects," Frontiers in Heat and Mass Transfer, 14, doi:10.5098/hmt.14.13. 